ساخت و بر رسى ساختار بلورى -مولكولى و طيفى يك كميلكس ليگاند-مخلوط جديد از مس(II) با ليعاند دو دندانه او •ا-فنانترولين به همراه ساليسيل آلدهيد S-S.بنزيل ايزوتيوسمى كربازون

\author{
ميلاد محجوبىزاده'، رضا تكجو"' زول ت. معَ

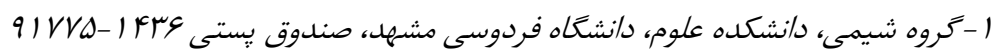

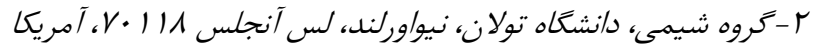

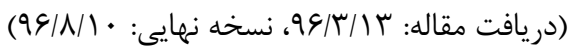

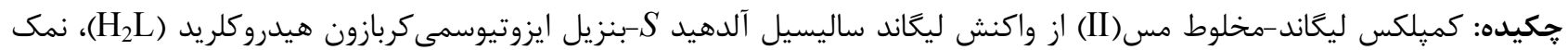

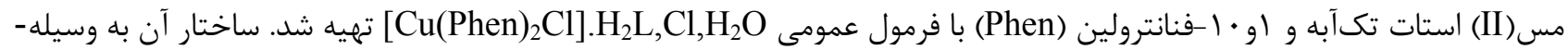

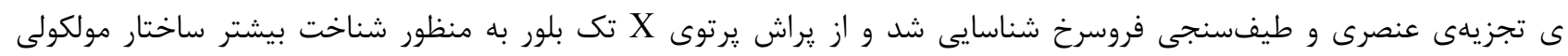

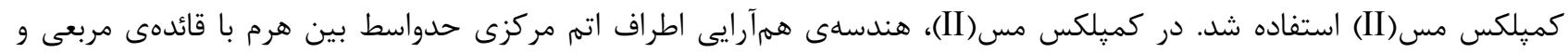
دوهرمى مثلثى است. دو ليعاند او··ا-فنانترولين (دهندهى NN) به صورت دو دندانهى خنثى، به فلز مركزى همتافته شده است. جايكاه ينجم با اتم كلر اشغال شده است.

وازههاى كليدى: باز شيف؛ /يزوتيوسمى كربازون؛ كميلكس مس(II)؛ ساختار بلورى؛ او. ا -فنانترولين.

ضد باكترى، ضد قارج و ضد سرطان دارند [11]. همجنين

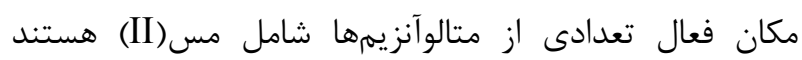

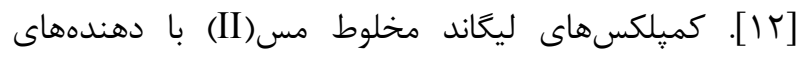

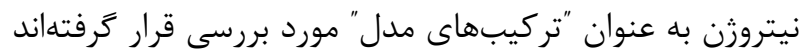

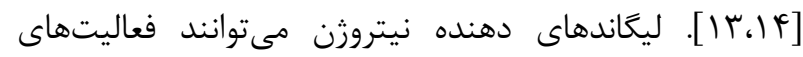

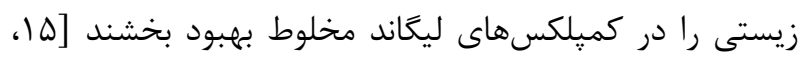

[19

واكنش تيوسمىكربازيد با آلكيل هاليدها، ايزوتيوسمى-

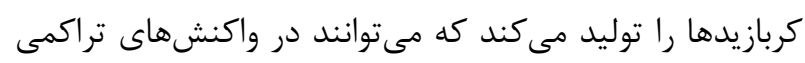

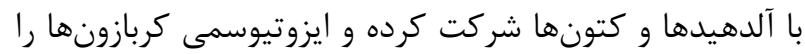

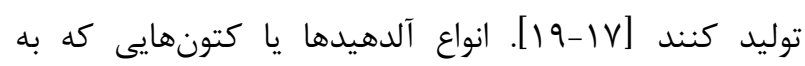

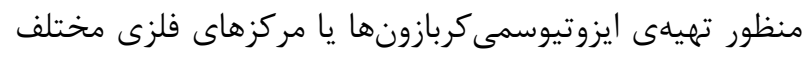

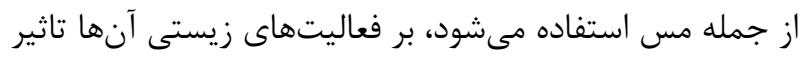

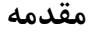

از جشمانداز زيست معدنى، كميلكسهاى مس بهد مدليل شباهت

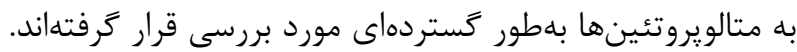
اين تركيبهاى مس، بهعنوان مكانهاى فعال زيستى بسيار

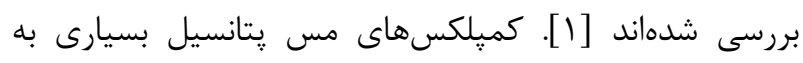

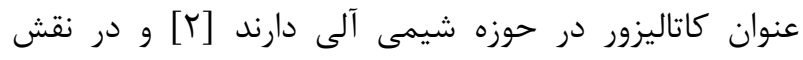

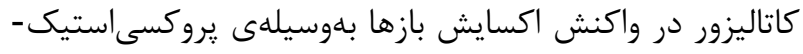

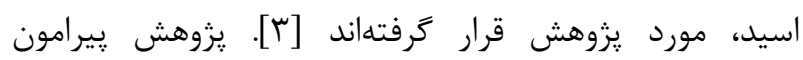

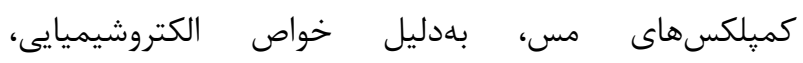

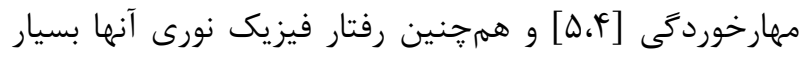

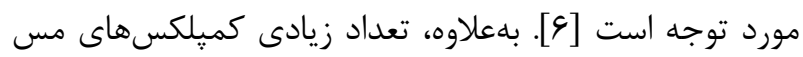

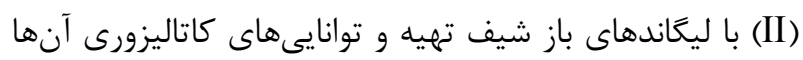

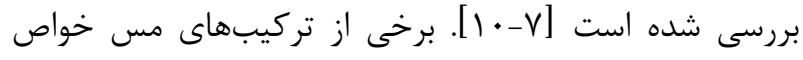


محيط سرد شد و سيس از محلول واكنش براى بلوركيرى اقدام

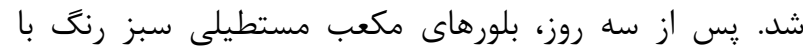

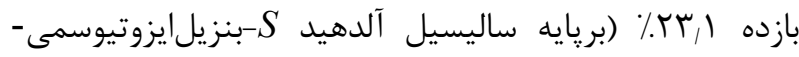

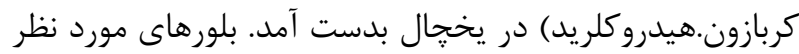
جداسازى شده و در محيط خشك شريد شدرند.

روشهاى مشخصه يابى

طيف فروسرخ اين تركيب با دستكاه طيفسنج مدل KBr در كسترعى Bock 500

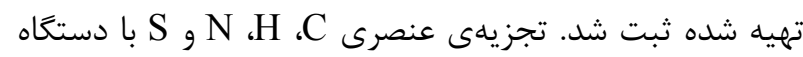
1112EA ساخت شركت Thermo Finnigan آمريكا، انجام شد. بهوسيلهى دستكاه جفت شده Bruker Smart APEX با تابش A (CCD)

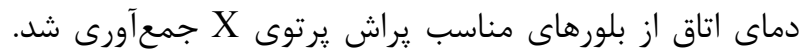

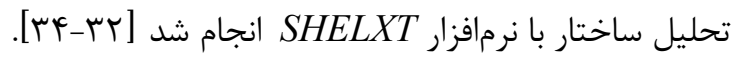
تجزيهى عنصرى براى محاسبه شده: كربن، هد

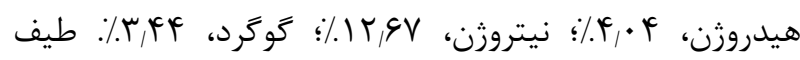

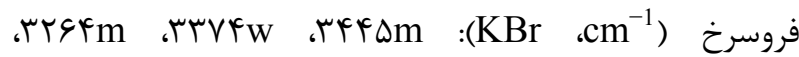

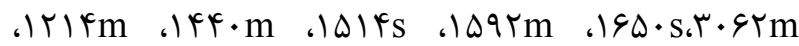

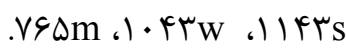

نتايج و بحث

از تقطير S- بنزيل ايزوتيوسمى كربازون.هيدروكلريد (Ha)

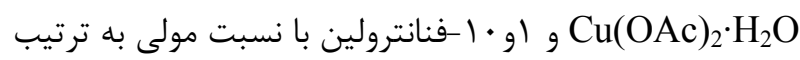

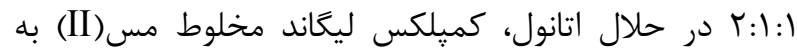

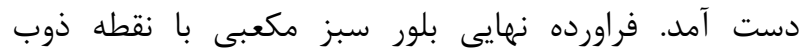

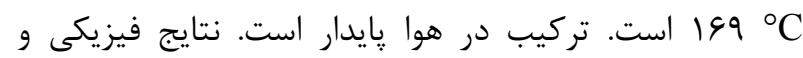

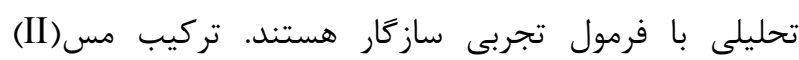

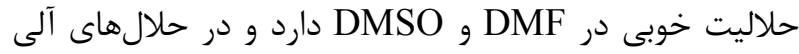
رايج مثل متانول، استون، كلرفرم و دى اتيل اتر،

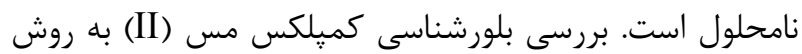
يراش يرتوى X نشان مى لنهد كه ساختار ماده با ساختار

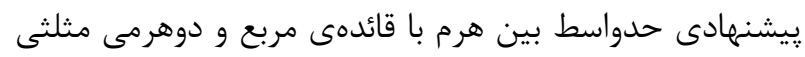

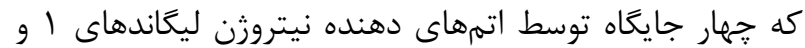

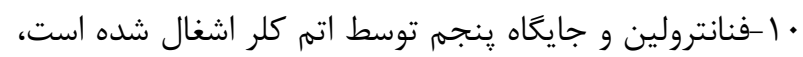

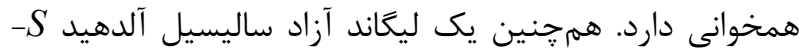

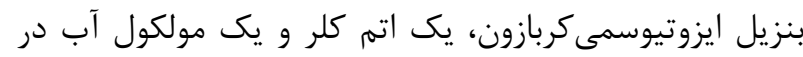

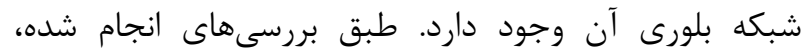
مراحل سنتز اين كميلكس به صورت شكل إست است بردي
مى كذارد [ ·r]. بهطور كلى، ايزوتيوسمى كربازيدها و مشتقهاى

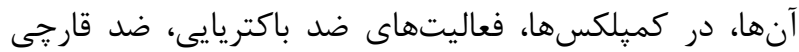

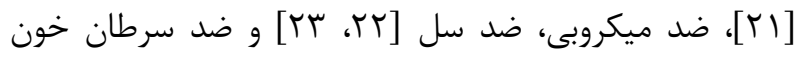

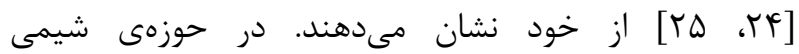

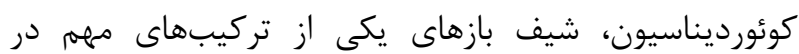

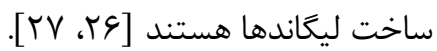

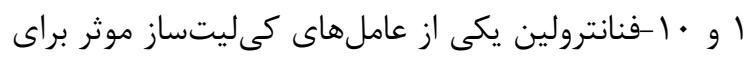

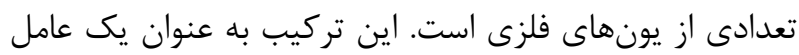
كميلكس

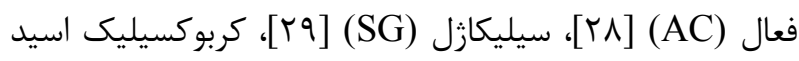

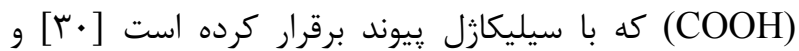
آلومينا [ابس] استفاده مىشود.

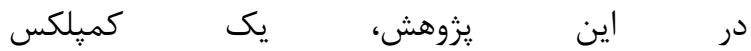
تجزئ تجزيه عنصرى و طيف فروسرخ شناسايى شد. همرجنين ساختار حالت جامد كميلكس مس(II) بررسى گرديد (شماى (). روشهاى تجربى مواد شيميايى مورد نياز در اين يزوهش با خلوص بالا خريدارى و بدون خالص سازى بيشتر استفاده شدند.

سنتز تركيب S-بنزيل ايزوتيوسمى كربازون.هيدروزنكلريد

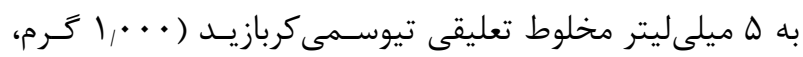

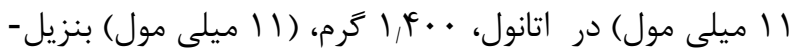

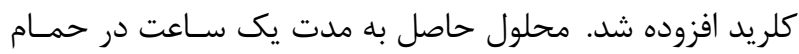

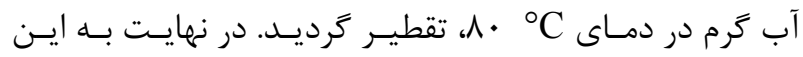

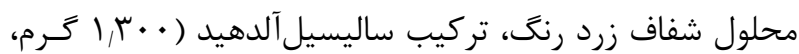

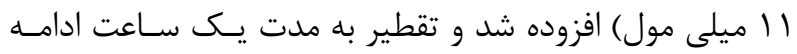

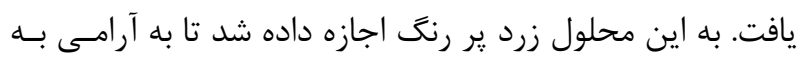

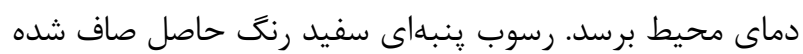
و با اتانول سرد شسته شد. فراورده در دسيكاتور حاوى سليك سليكازل در شرايط خلاء خشك ترديد.

\section{سنتز تركيب} • ا ميلىليتر مخلوط تعليقى اتانولى سبز رنغ از ليكاند

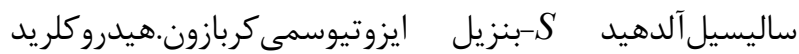

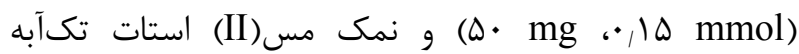

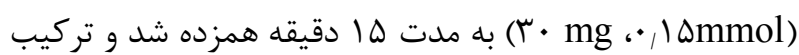

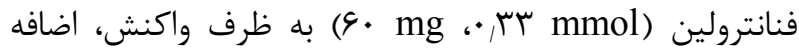

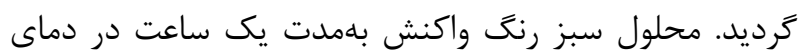

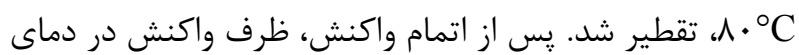




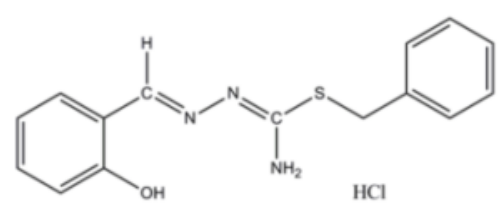

$+\mathrm{Cu}(\mathrm{OAC})_{2} \cdot \mathrm{H}_{2} \mathrm{O}+$
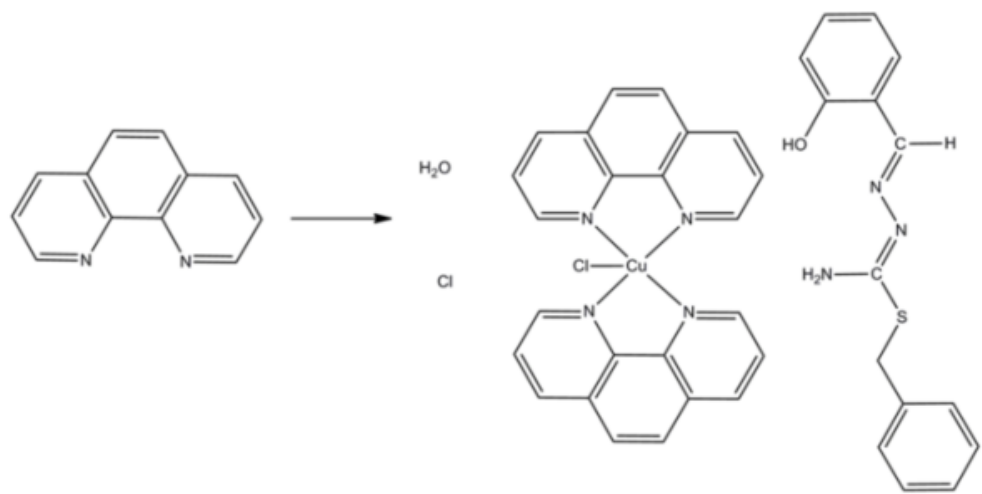

شماى 1 مراحل تهيهى كميلكس

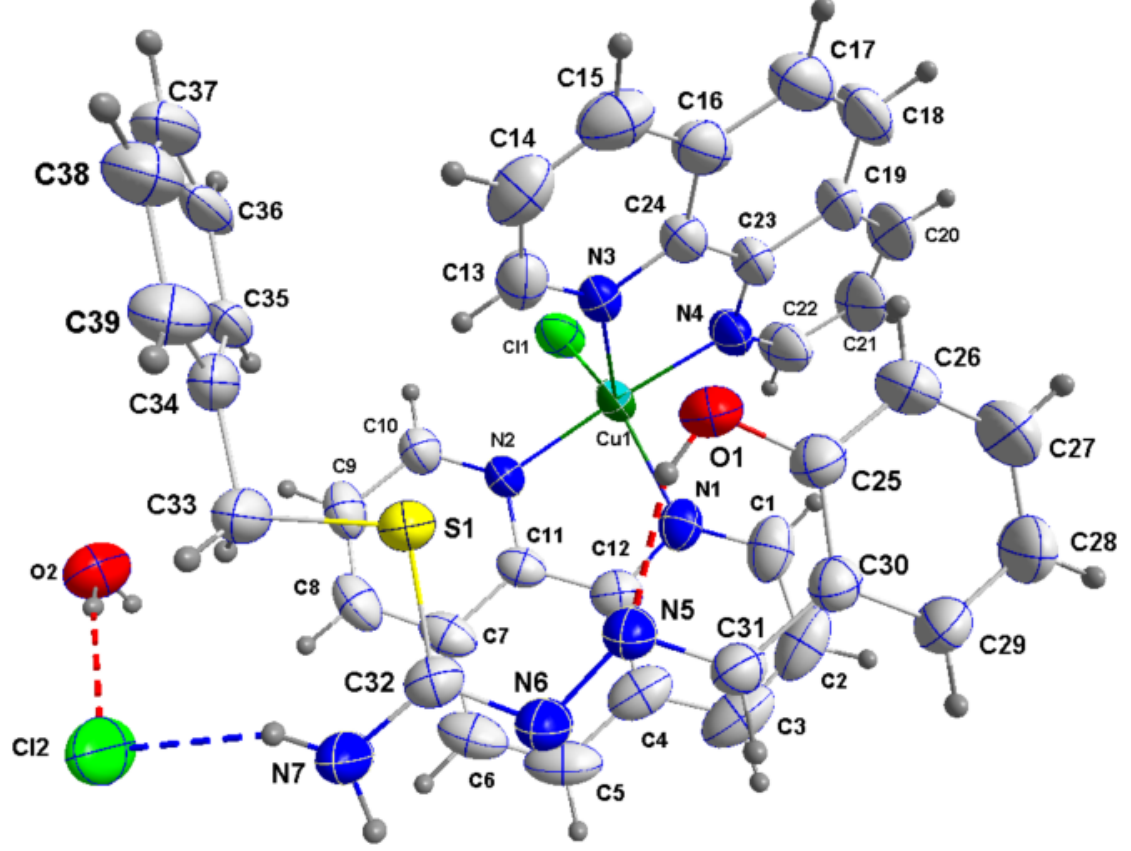

شكل ا نمايى از تركيب

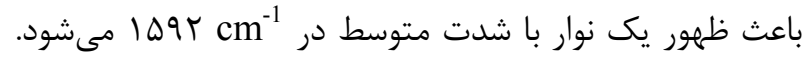

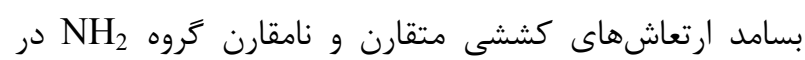

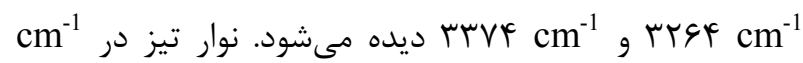

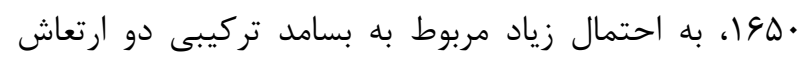

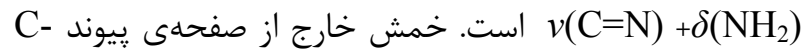
قابل مشاهده است. نوار جذبى مشاهده شده

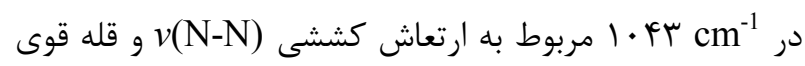

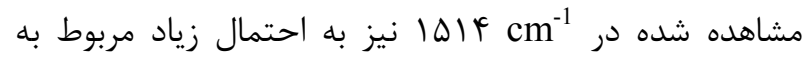
تركيب ارتعاشهاى بسامد كششى مربوط به كروه ليكاند

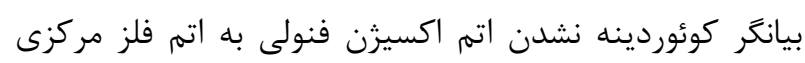

بررسى طيف فروسرخ

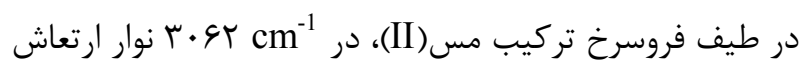

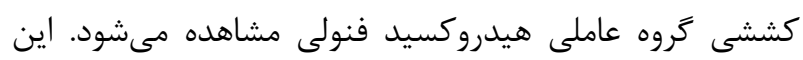

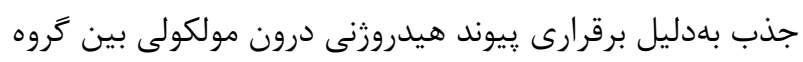

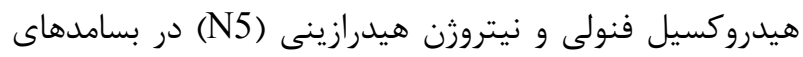

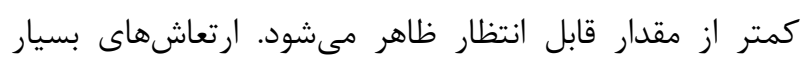

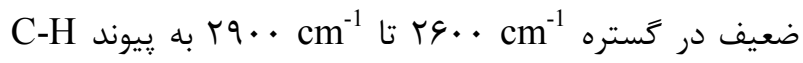

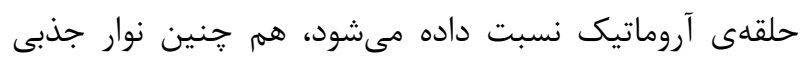

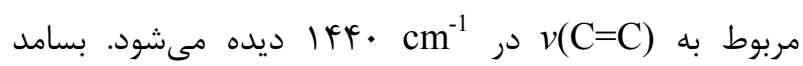

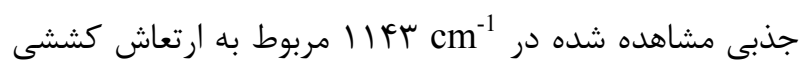

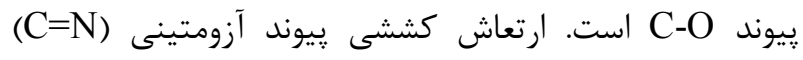


جدول ا آورده شده است. طول و زاويه پِيوندهاى انتخاب شده

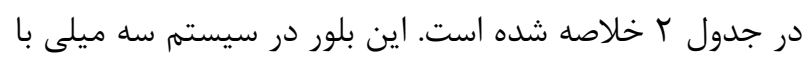

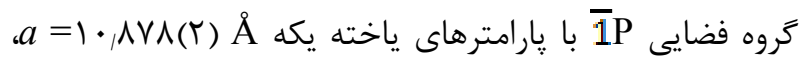
$، \alpha=q \Lambda, \Gamma \wedge \&(\Gamma)^{\circ} c c=19, \Gamma \& \vee(\Gamma) \AA ، b=11, \uparrow \Delta \vee(\Gamma) \AA$

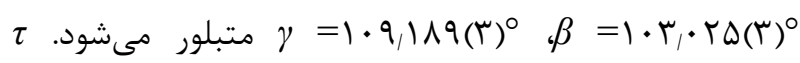

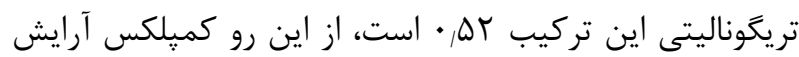

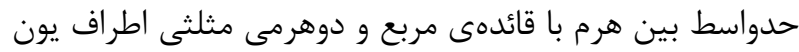

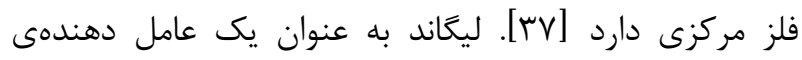

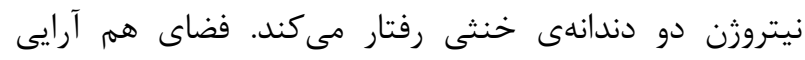

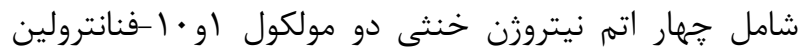
است. جايكاه باقيمانده ساختار توسط اتم كلر اشغال مى نى دود.
است. انرزى مدهاى حلقه او.|-فنانترولين (CC) $\mathrm{cm}^{-1}$ ، $1419 \mathrm{~cm}^{-1}$ ، $1 \Delta \cdot \Delta \mathrm{cm}^{-1}$ ، I Y $\cdot \mathrm{cm}^{-1}$ د ( $(\mathrm{CN})$

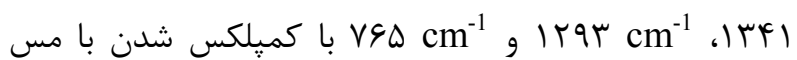

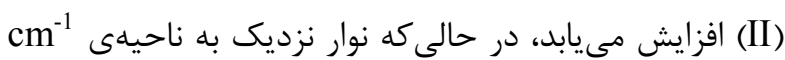

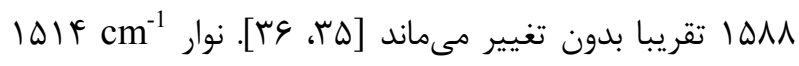
به ارتعاش تنفسى ييريدين نسبت داده مىشود. نوار مشاهده

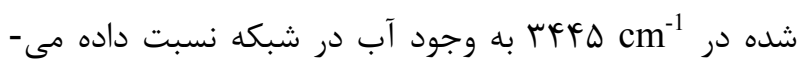

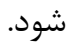

بر سلى ساختار بلورى تركيب Cu(Phen)

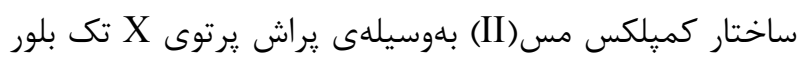

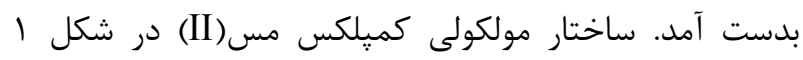
نشان داده شده است. دادهاى بلورشناسى اين تركيب در

جدول ا دادهاى بلورشناسى مربوط به تركيب

\begin{tabular}{|c|c|}
\hline فرمول & $\mathrm{C}_{\Gamma \varphi} \mathrm{H}_{\mu \Gamma} \mathrm{Cl}_{\Gamma} \mathrm{CuN}_{r} \mathrm{O}_{r} \mathrm{~S}$ \\
\hline $\mathrm{F}_{\mathrm{w}}$ & $V 9 \Lambda, r T$ \\
\hline$a(\AA)$ & $1 \cdot \wedge \vee \wedge(Y)$ \\
\hline$b(\AA)$ & $11, F \Delta \vee(r)$ \\
\hline$c(\AA)$ & $19, \Gamma \& \vee(r)$ \\
\hline$\alpha(\operatorname{deg})$ & $9 \wedge, \Gamma \wedge \&(\uparrow)$ \\
\hline$\beta(\operatorname{deg})$ & $1 \cdot r \cdot r \Delta(r)$ \\
\hline$\gamma(\operatorname{deg})$ & $1 \cdot 9,119(\pi)$ \\
\hline space group & $\mathrm{P} \overline{1}$ \\
\hline $\mathrm{D}_{\text {calcd }}\left(\mathrm{g}_{/} \mathrm{cm}^{3}\right)$ & $1,4 \& \pi$ \\
\hline Z & f \\
\hline crystal system & 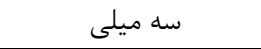 \\
\hline volume $\left(\AA^{3}\right)$ & $|\wedge| r, r(V)$ \\
\hline $\mathrm{F}(000)$ & NTr \\
\hline ضر يب جذب (mm-1) & $\cdot \wedge \Delta F$ \\
\hline Reflns .collected & IVTME \\
\hline unique reflns. & $\Lambda \vee \Delta \cdot$ \\
\hline شاخص نيكويى برازش & $1, \cdots$ \\
\hline$R_{\text {int }}$ & $\cdot \cdot r t \cdot$ \\
\hline$R_{1}[\mathrm{I}>2 \sigma(\mathrm{I})]$ & $\cdot .+P V I$ \\
\hline$w R_{2}[\mathrm{I}>2 \sigma(\mathrm{I})]$ &.$/ 1 \mathrm{KV}$ \\
\hline $\mathrm{R}_{1}$ (all data) & $\cdot \cdot \wedge \Delta \varphi$ \\
\hline$w \mathrm{R}_{2}$ (all data) & $\cdot 1 / Y 49$ \\
\hline largest diff. peak and hole $\left(\mathrm{e} \AA^{-3}\right)$ & $\cdot \Delta \cdot \cdot,-\cdot,|\cdot| \cdot$ \\
\hline
\end{tabular}




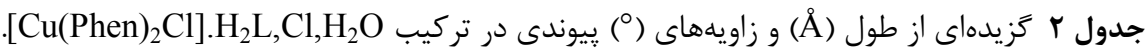

\begin{tabular}{|c|c|c|c|}
\hline \multicolumn{2}{|c|}{ § طول يیوند } & \multicolumn{2}{|c|}{ (") زاويه يیيوند } \\
\hline Cu1-N1 & $r_{1} \cdot \wedge \vee(r)$ & N1-Cu1-N4 & $૧ \uparrow, \wedge F(१)$ \\
\hline $\mathrm{Cu} 1-\mathrm{N} 2$ & 1,99\%r(19) & N4-Cu1-N3 & $\vee 9,9 \vee(\curlywedge)$ \\
\hline $\mathrm{Cu} 1-\mathrm{N} 3$ & $r, 19 V(r)$ & N3-Cu1-N2 & $q \vee, \wedge r(\Lambda)$ \\
\hline $\mathrm{Cu} 1-\mathrm{N} 4$ & $1,991(T)$ & N2-Cu1-N1 & $\wedge 1, \cdot 1(\wedge)$ \\
\hline \multirow[t]{4}{*}{$\mathrm{Cu} 1-\mathrm{Cl1}$} & T,TAT·(Q) & N1-Cu1-Cl1 & $|f|, \Delta q(\xi)$ \\
\hline & & N4-Cu1-Cl1 & $9 T, \Lambda)(V)$ \\
\hline & & N3-Cu1-Cl1 & $1 \cdot 9, \Delta \mid(9)$ \\
\hline & & N2-Cu1-Cl1 & $9 \%, 9 \Delta(9)$ \\
\hline
\end{tabular}

نشان داده شده است. اين يديده ممكن است بهدليل آثار

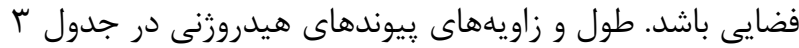
ارائه شده است. در واحد بى تقارن اين تركيب، شش برهم كنش

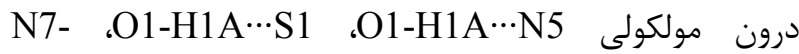

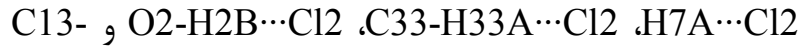

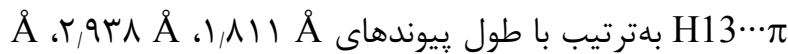
蓜 r) symmetry code $i$ : 1-x,1-y,-z; ii: 1-) N7-H7B $\cdots \mathrm{N}^{\mathrm{ii}}$

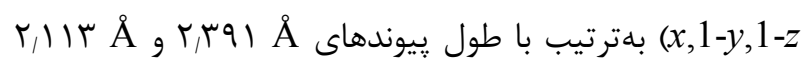

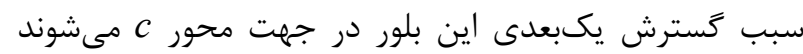

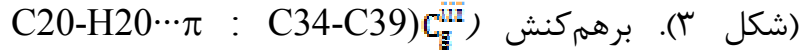

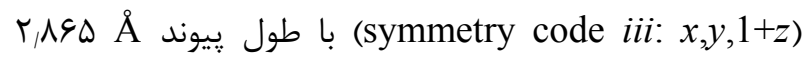
سبب رشد دوبعدى اين بلور در جهت محور a مى

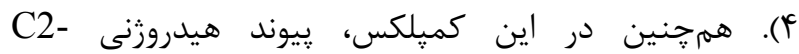
(symmetry code $i v: 1+x, 1+y, z) \quad \mathrm{H} 2 \cdots \mathrm{Cl}^{i v}$

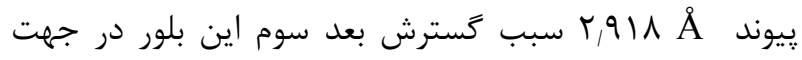
محور b مئشود (شكل ه).
ييوندهاى دهندهى مس Cu1-N1، Cu1-N2، Cu1-Cl1 و Cu1-N4

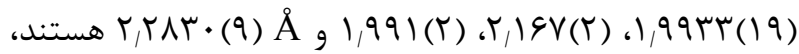
كه توسط مقادير مشاهده شده براى اين دسته از كميلكسهاى (1)

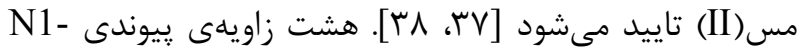
،N2-Cu1-N1 ،N3-Cu1-N2 ،N4-Cu1-N3 ،Cu1-N4 N2-Cu1- , N3-Cu1-Cl1 ،N4-Cu1-Cl1 ،N1-Cu1-Cl1 Cl1

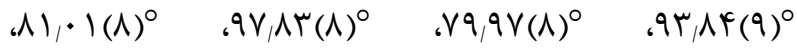
. اختلاف معنادار بين دو زاويهى N2-Cu1-N1 N4-Cu1-N3 و دو زاويه N1-Cu1-N4، N3-Cu1-N2 به دليل تشكيل حلقهى ينج عضوى كى ليت است. اين مشاهده براى كميلكس -

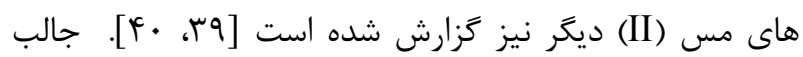
اين است كه اتم كلر به اتم N4 حلقه او.ا-فنانترولين نزديكتر است، جنان كه با مقايسه زاويهى N4-Cu1-Cl1

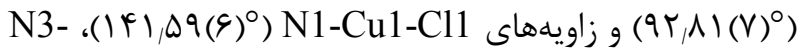
$\left.\left(9 \mathrm{r}, 9 \Delta(9)^{\circ}\right) \mathrm{N} 2-\mathrm{Cu} 1-\mathrm{Cl} 1,(1 \cdot 9,0)(9)^{\circ}\right) \mathrm{Cu} 1-\mathrm{Cl} 1$

جدول r جزييات ييوند هيدرورنى (طول يِيوند بر حسب §ُ).

\begin{tabular}{|c|c|c|c|c|}
\hline $\mathrm{D}-\mathrm{H} \cdots \mathrm{A}$ & $\mathrm{d}(\mathrm{D}-\mathrm{H})$ & $\mathrm{d}\left(\mathrm{H} \cdots \cdots^{\mathrm{A}}\right)$ & $\mathrm{d}(\mathrm{D}-\mathrm{A})$ & $<(\mathrm{DHA})$ \\
\hline $\mathrm{O} 1-\mathrm{H} 1 \mathrm{~A} \cdots \mathrm{S} 1$ & $\cdot \wedge V$ & $r, 94$ & $r, s \mid v(r)$ & Tra, \\
\hline O1-H1A $\cdots$ N5 & $\cdot \wedge v$ & 1,11 & $r, 9 \mid r(r)$ & $1 Q T_{.}$. \\
\hline $\mathrm{N} 7-\mathrm{H} 7 \mathrm{~A} \cdots \mathrm{Cl}^{2}$ & .99 & $r, r q$ & $r, r \Delta F(r)$ & $\mid \Delta V,{ }^{F}$ \\
\hline N7-H7B...N6 & .91 & $r, 11$ & $r, \cdot 1 r(r)$ & 199,9 \\
\hline $\begin{array}{l}\mathrm{O} 2-\mathrm{H} 2 \mathrm{~B} \cdots \boldsymbol{C C l} \\
\mathrm{O} 2-\mathrm{H} 2 \mathrm{~A} \cdots \mathrm{Cl}^{2}\end{array}$ & $\begin{array}{l}\cdot \wedge V \\
\cdot \wedge V\end{array}$ & $\begin{array}{l}r, T \Delta \\
r, F,\end{array}$ & $\begin{array}{l}r, r \cdot r(r) \\
r, r r r(r)\end{array}$ & $\begin{array}{l}19 V, 1 \\
1 V G, V\end{array}$ \\
\hline
\end{tabular}




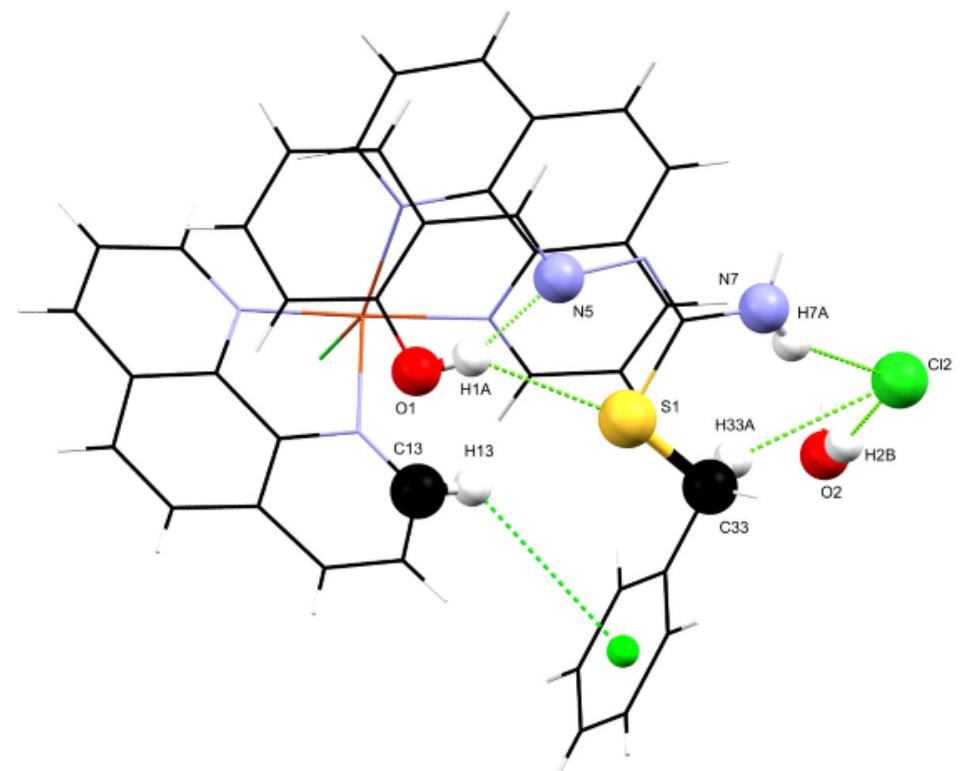

شكل r برهم كنشهاى درون مولكولى در تركيب

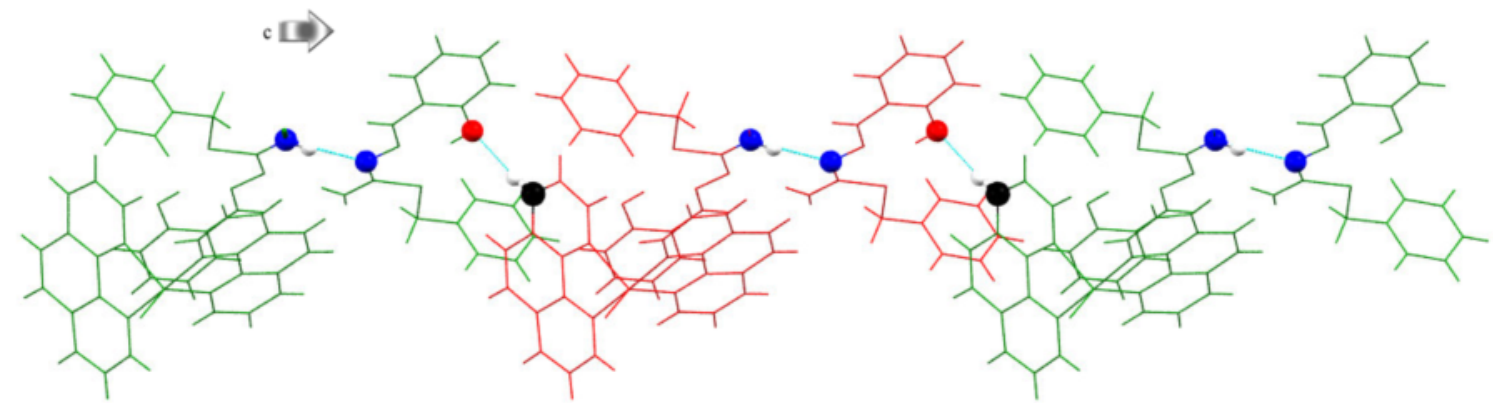

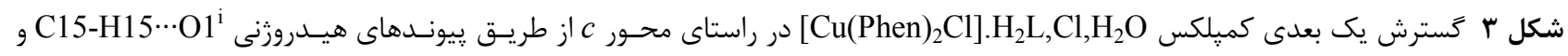
.(symmetry code $i: 1-x, 1-y,-z ; i i$ : $1-x, 1-y, 1-z) \mathrm{N} 7-\mathrm{H} 7 \mathrm{~B} \cdots \mathrm{N} 6^{\mathrm{ii}}$

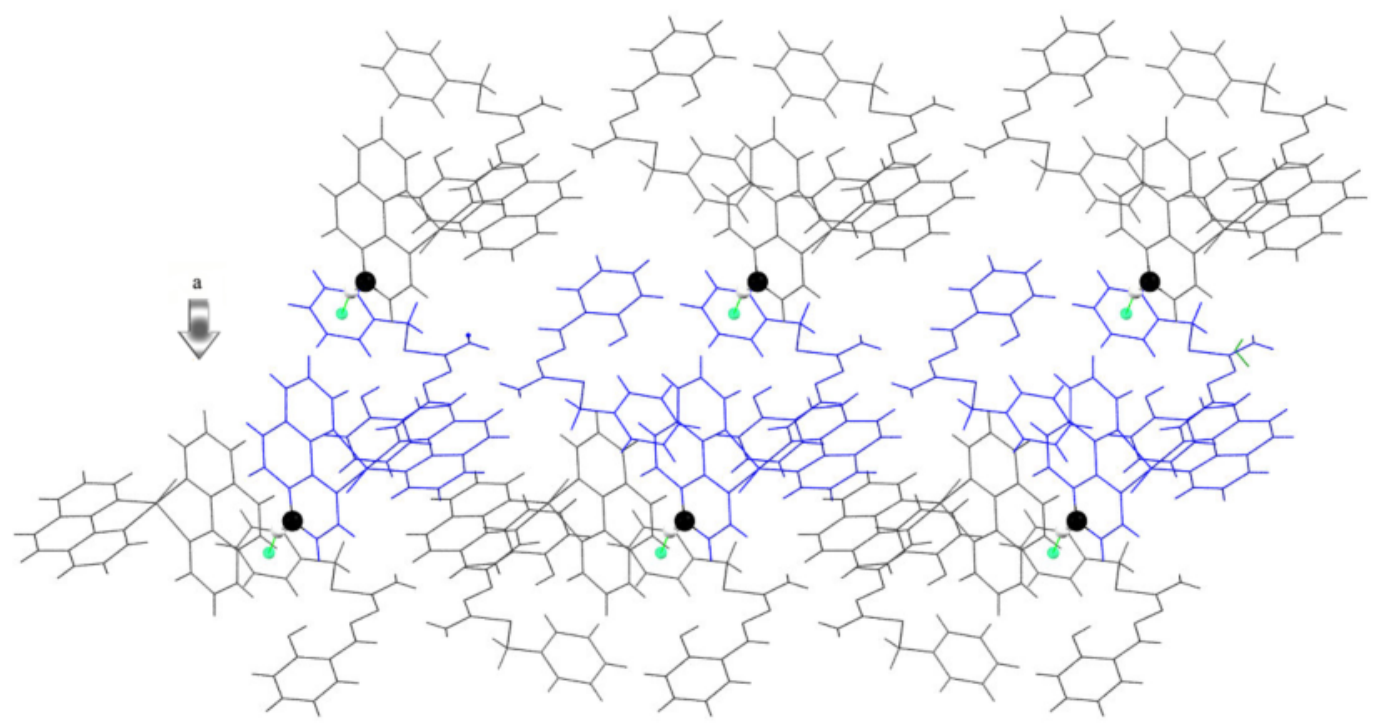

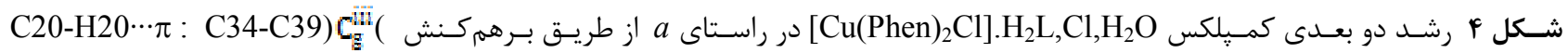
(symmetry code iii: $x, y, 1+z$ ) 


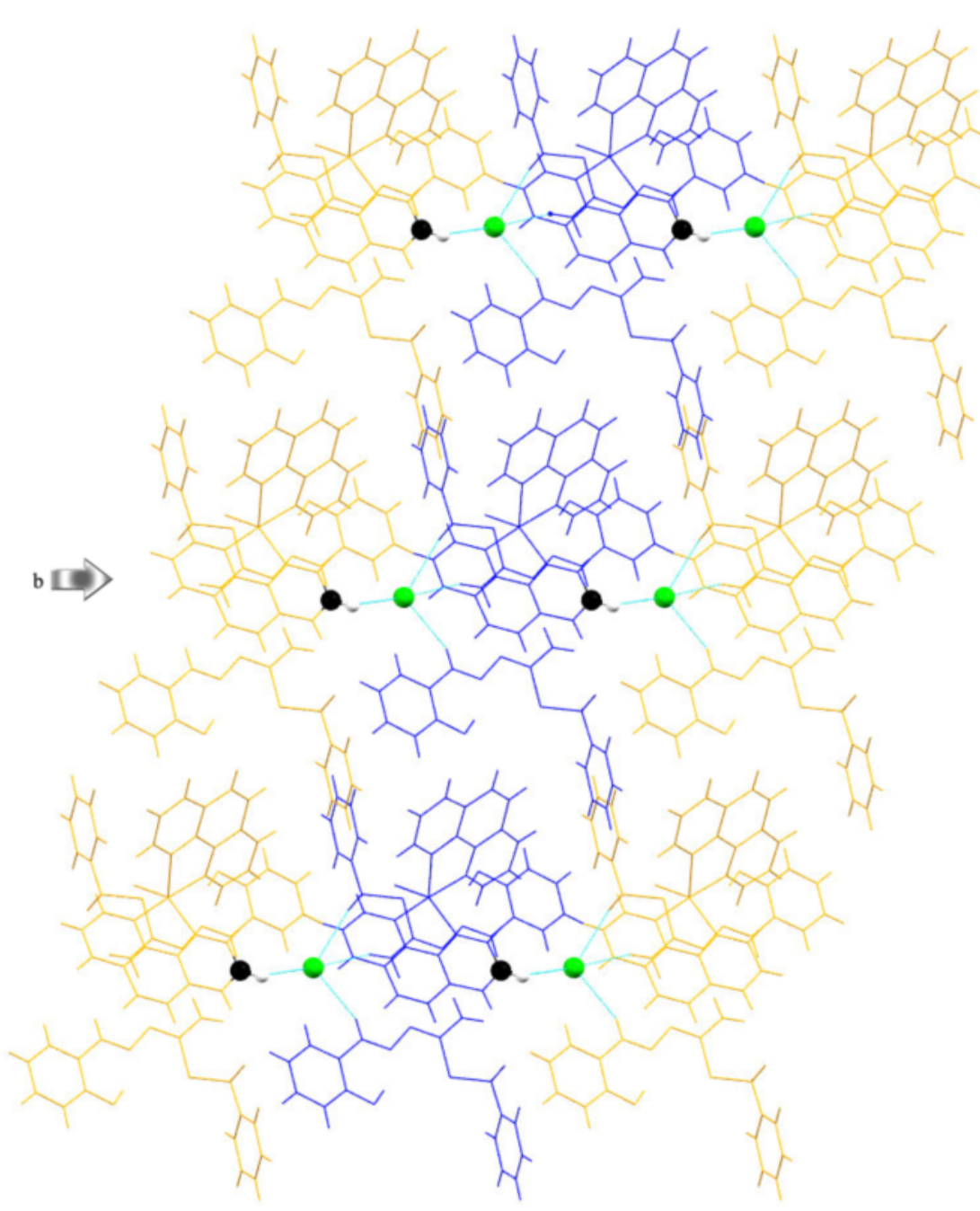

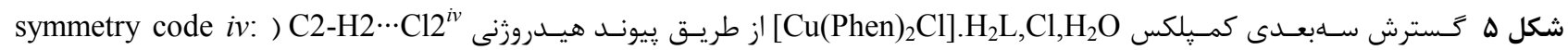
. $(1+x, 1+y, z$

ينج كوئوردينه حدواسط بين هرم با قائدهى مربع و دوهرمى مثلثى را براى مس نشان مىدهند. همرجنين شش برهمكنش

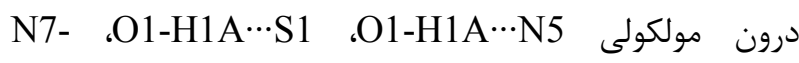

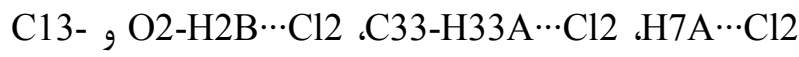
د13 در واحد بعتقارن اين تركيب وجود دارد. در اين

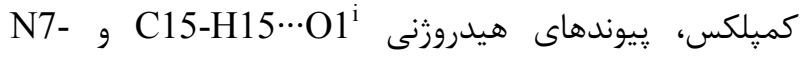
symmetry Code $i$ : $1-x, 1-y,-z ; i i: 1-x, 1-)$ H7B $\cdots \mathrm{N} 6^{\mathrm{ii}}$ (y,1-z موجب رشد يكبعدى اين تركيب مىشوند. همرجنين

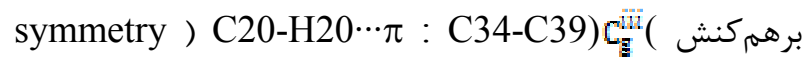
(code iii: $x, y, 1+z$
برداشت يك ليخاند دهندهى نيتروزن متقارن (Phen) و كميلكس مس آن با تركيب (II) كميلكس فلزى بلوسيله تجزيهى عنصرى و طيفسنجى فروسرخ شناسايى شد. بهعلاوه، يراش يرتوى X براى شناسايى كميلكس فلزى انجام شد. تجزيههاى عنصرى كربن، هيدروزن، نيتروزن و گو بلورشناسى گيرتوى X نتيجه گرفته مىشود كه هر يك از دو ليعاند او، ا-فنانترولين به عنوان يك كىليت دو دندانهى خنثى از طريق دو اتم نيتروزن به فلز مركزى و همرجنين يك اتم كلر به فلز مركزى كوئوردينه شده است. نتايج يك تركيب 
towards molecular oxygen", Synthetic Metals 161 (2011) 1377-1382.

[7] Pardey A.J., Rojas A.D., Yánez J.E., Betancourt P., Scott C., Chinea C., Urbina C., Moronta D., Longo C., "Spectroscopic characterization of coordination complexes based on dichlorocopper(II) and poly(4-vinylpyridine): Application in catalysis", Polyhedron 24 (2005) 511-519.

[8] Hakimi M., Vahedi H., Takjoo R., Rezaeifard A., "Nanoporous Silica Supported Novel Copper (II) Thiosemicarbazone Complexes as Selective and Reusable Catalysts for Oxidation of Alcohols Using $\mathrm{H}_{2} \mathrm{O}_{2}$ ", International Journal of ChemTech Research 4 (2012)1658-1665.

[9] Hu Z., Kerton F.M., "Room temperature aerobic oxidation of alcohols using $\mathrm{CuBr} 2$ with TEMPO and a tetradentate polymer based pyridyl-imine ligand", Applied Catalysis A: General 413-414 (2012) 332-339.

[10] Heshmatpour F., Rayati S., Afghan H.M., Abdolalian P., Neumueller B., "Copper(II) Schiff base complexes derived from 2,2'-dimethylpropandiamine: Synthesis, characterization and catalytic performance in the oxidation of styrene and cyclooctene", Polyhedron 31(2012) 443-450.

[11] Wang Q., Bi C.F., Fan Y.H., Zhang X., Zuo J., Liu S.B., "A novel copper(II) complex with Schiff base derived from o-vanillin and $L$ methionine: Syntheses and crystal structures", Russian Journal of Coordination Chemistry 37 (2011) 228-234.

[12] Arion V.B., Rapta P., Telser J., Shova S.S., Breza M., Lušpai M., J. Kožišek. "Syntheses, Electronic Structures, and EPR/UV-Vis-NIR Spectroelectrochemistry of Nickel(II), Copper(II), and Zinc(II) Complexes with a Tetradentate Ligand Based on

S-Methylisothiosemicarbazide”, Inorganic Chemistry 50 (2011) 2918-2931.

[13] Tong M.L., Li L.J., Mochizuki K., Chang H.C., Chen X.M., Kitagawa S., "A novel threedimensional coordination polymer constructed with mixed-valence dimeric copper(I,II) units", Chemical Communications (2003) 428-429.

[14] King P.B., Encyclopedia of Inorganic Chemistry, John Wiley \& Sons, New York, NY (1994) 829.

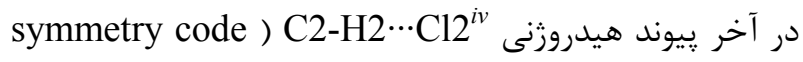
(iv: 1+x,1+y,z سبب گسترش سه بعدى اين بلور مىشود.

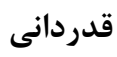
نويسندگان اين مقاله، مراتب قدردانى خود را از حمايتهاى

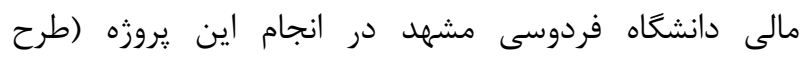

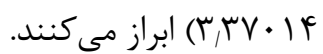
كد laff.q P CCDC حاوى دادهاى بلورشناسى براى اين

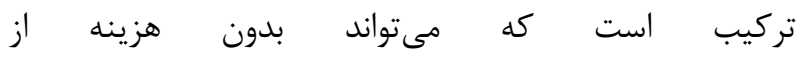


[1] Cotelesage J.J.H., Pushie M.J., Grochulski P., Pickering I.J., George G.N., "Metalloprotein active site structure determination: Synergy between X-ray absorption spectroscopy and X-ray crystallography", Journal of Inorganic Biochemistry 115 (2012) 127-137.

[2] Pathan N.B., Rahatgaonkar A.M., Chorghade M.S., "Metal-organic framework $\mathrm{Cu}_{3}(\mathrm{BTC})_{2}\left(\mathrm{H}_{2} \mathrm{O}\right)_{3}$ catalyzed Aldol synthesis of pyrimidine-chalcone hybrids", Catalysis Communications 12 (2011) 1170-1176.

[3] Gradinaru J., Forni A., Simonov Y., Popovici M., Zecchin S., Gdaniec M., Fenton D.E., "Mononuclear nickel(II) and copper(II) complexes with Schiff base ligands derived from 2,6-diformyl4-methylphenol and $S$ methylisothiosemicarbazones", Inorganica Chimica Acta 357 (2004) 2728-2736.

[4] Naskar J.P., Guhathakurta B., Lu L., Zhu M., "Oximato bridged dinuclear copper(II) complexes: Synthesis, crystal structure, magnetic, thermal, electrochemical aspects and BVS analysis", Polyhedron 43 (2012) 89-96.

[5] Aytaç A., " $\mathrm{Cu}(\mathrm{II}), \mathrm{Co}(\mathrm{II})$ and $\mathrm{Ni}(\mathrm{II})$ complexes of $-\mathrm{Br}$ and $-\mathrm{OCH}_{2} \mathrm{CH}_{3}$ substituted Schiff bases as corrosion inhibitors for aluminum in acidic media", Journal of Materials Science 45 (2010) 6812-6818.

[6] Jia J., Tian Y., Li Z., "Mesoporous silica doped with a chemosensor based on phosphorescent copper(I) complex: Synthesis, characterization, photophysical property, and sensing behavior 
[24] Atasever B., Ulküseven B., Bal-Demirci T., Erdem-Kuruca S., Solakoğlu Z., "Cytotoxic activities of new iron(III) and nickel(II) chelates of some S-methyl-thiosemicarbazones on K562 and ECV304 cells", Investigational New Drugs 28 (2010) 421-432.

[25] Belicchi-Ferrari M., Bisceglie F., Casoli C., Durot S., Morgenstern-Badarau I., Pelosi G., Pilotti E., Taras-coni P., "Copper(II) and Cobalt(III) Pyridoxal Thiosemicarbazone Complexes with Nitroprusside as Counterion: Syntheses, Electronic Properties, and Antileukemic Activity", Journal of Medicinal Chemistry 48 (2005) 16711675.

[26] Makode J.T., Yaul A.R., Bhadange S.G., Aswar A.S., "Physicochemical characterization, thermal, and electrical conductivity studies of some transition metal complexes of bis-chelating Schiff base", Russian Journal of Inorganic Chemistry 54 (2009) 1372-1377.

[27] Takjoo R., Ramsami P., Mague J., Hasani Z., Rhyman L., "Dioxidomolybdenum(VI) complexes of allyl N'-2-hydroxy-3methoxybenzylidenecarbamohydrazonothioate:

Synthesis, spectral, and theoretical investigations", Journal of Coordination Chemistry 69 (2016) 1100-1109.

[28] Mikula B., Puzio B., "Determination of trace metals by ICP-OES in plant materials after preconcentration of 1,10-phenanthroline complexes on activated carbon", Talanta, 71 (2007) 136-140.

[29] Mikula B., Puzio B., \& Feist B., "Preconcentration of $\mathrm{Cd}(\mathrm{II}), \mathrm{Pb}(\mathrm{II}), \mathrm{Co}(\mathrm{II}), \mathrm{Ni}(\mathrm{II})$, and $\mathrm{Cu}(I I)$ by solid-phase extraction method using 1,10-phenanthroline", Journal of Analytical Chemistry 64 (2009) 786-790.

[30] Mikula B., Puzio B., Feist B., "Application of 1,10-phenanthroline for preconcentration of selected heavy metals on silica gel", Microchimica Acta 166 (2009) 337-341.

[31] Shabani A.M.H., Dadfarnia S., Dehghani Z., "On-line solid phase extraction system using 1,10phenanthroline immobilized on surfactant coated alumina for the flame atomic absorption spectrometric determination of copper and cadmium”, Talanta 79 (2009) 1066-1070.
[15] Feng-Tai Chen C., Tang G.-R., Jin G.-X., "Novel nickel(II) and copper(II) complexes with phenoxy-imidazole ligands: Syntheses, crystal structures and norbornene addition polymerization", Journal of Organometallic Chemistry 692 (2007) 3435-3442.

[16] Takjoo R., Mague J., Akbari A., Ebrahimipour S.Y., "Synthesis, structural, and thermal analyses of copper(II) and oxidovanadium(IV) complexes of 4-bromo-2-(((5chloro-2-hydroxyphenyl)imino)methyl)phenol",

Journal of Coordination Chemistry 66 (2013) 2852-2862.

[17] Kurt Y.d., Ulküseven B., Tuna S., Ergüven M., Solakoğlu S., "Iron(III) and nickel(II) template complexes derived from benzophenone thiosemicarbazones", Journal of Coordination Chemistry 62 (2009) 2172-2181.

[18] Kurt Y.D., Ulküseven B., “Unusual template condensation of benzophenone thiosemicarbazones and salicylaldehydes with nickel(II)", Journal of Coordination Chemistry 63 (2010) 828-836.

[19] Refat M.S., El-Deen I.M., Anwer Z.M., ElGhol S., "Spectroscopic studies and biological evaluation of some transition metal complexes of Schiff-base ligands derived from 5-arylazosalicylaldehyde and thiosemicarbazide", Journal of Coordination Chemistry 62 (2009) 1709-1718.

[20] Beraldo H., Gambino D. "The wide pharmacological versatility of semicarbazones, thiosemicarbozones and their metal complexes", Mini-Reviews in Medicinal Chemistry 4 (2004) 31.

[21] Kizilcikli İ., Kurt Y.D., Akkurt B., Genel A.Y., Birteksz S., Ulküseven B., "Antimicrobial activity of a series of thiosemicarbazones and their ZnII and PdII complexes", Folia Microbiologica, 52 (2007) 15-25.

[22] Cocco M.T., Congiu C., Onnis V., Pellerano M.L., Logu A. De., "Synthesis and antimycobacterial activity of new $S$ alkylisothiosemicarbazone derivatives", Bioorganic and Medicinal Chemistry, 10 (2002) 501-506.

[23] Amit N., Rahul J., "Recent Advances in New Structural Classes of Anti-Tuberculosis Agents", Current Medicinal Chemistry 12 (2005) 18731886. 
methylbenzimidazol-2'-yl)-2,6-dithiaheptane] copper (II) perchlorate", Journal of the Chemical Society, Dalton Transactions. (1984) 1349-1356.

[38] Galesic N., Trojko R., Leovac V.M., "Structure of (2,4-dihydroxybenzaldehyde Smethylisothiosemicarbazonato) isothiocyanato Copper(II)-dihydrate”, Zeitschrift Für Kristallographie 203 (1993) 57-66.

[39] Takjoo R., Akbari A., Ahmadi M., Amiri Rudbari H., Bruno G., "New 5-bromo-2hydroxybenzaldehyde S-ethylisothiosemicarbazone and its mixed-ligand $\mathrm{Cu}(\mathrm{II})$ complex with imidazole: synthesis, characterization and DFT calculation", Central European Journal of Chemistry (11) (2013) 1844-1851.

[40] Bourosh P.N., Zhovmir F.K., Simonov Y.A., Dvorkin A.A., Gerbeleu N.V., "Crystalline and molecular-structure of $\quad S$-methyl-N'-(3carboxysalicylidene) isothiosemicarbazidato$O, N^{l}, N^{4}(2-$-)-(ammine)

Copper(II)", Koordinatsionnaya Khimiya 19 (1993) 864-867.
[32] Bruker-AXS3, SADABS, SAINT, SHELXTL, Madison, WI, 2016.

[33] Sheldrick G. M., SHELXT, Acta Crystallographica Section A 71 (2015a) 3-8.

[34] Sheldrick G. M., SHELXL-2014, Acta Crystallographica Section C 71 (2015b) 3-8.

[35] Ueno K., Martell A.E., "Infrared study of metal chelates of bisacetylacetoneethylenediimine and related compounds", Journal of Physical Chemistry 59 (1955) 998-1004.

[36] Campos-Vallette M.M., Clavijo R.E., Mendizabal F., Zamudio W., Baraona Ruth, Diaz G., "Infrared spectrum of the bis-(1,10phenanthroline) $\mathrm{Cu}(\mathrm{I})$ and $\mathrm{Cu}(\mathrm{II})$ perchlorate complexes", Vibrational Spectroscopy 12 (1996) 37-44.

[37] Addison A. W., Rao N. T., Reedijk J., van Rijn J., Verschoor G. C., "Synthesis, structure, and spectroscopic properties of copper(II) compounds containing nitrogen-sulphur donor ligands; the crystal and molecular structure of aqua[1,7-bis(N- 